Pacific Journal of Mathematics

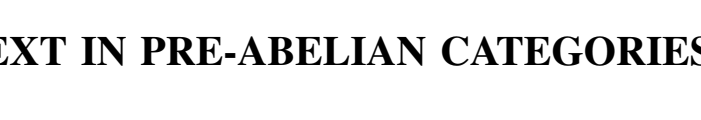




\title{
EXT IN PRE-ABELIAN CATEGORIES
}

\author{
Fred Richman and Elbert A. Walker
}

A natural definition for Ext is given in an arbitrary pre-abelian category. Ext is an additive bifunctor from the category to abelian groups, the five lemma and the $3 \times 3$ lemma hold, and $\mathrm{Ext}^{n}$ may be defined in the usual projectiveless way to yield the standard exact sequence of Ext's. Examples are given of what Ext is in various specific preabelian categories.

1. Semi-stable kernels and cokernels. Throughout this paper we shall be dealing with a pre-abelian category, that is, an additive category with kernels and cokernels. If $f: A \rightarrow B$ and $\xi: B^{\prime} \rightarrow B$, then we can complete the pullback diagram

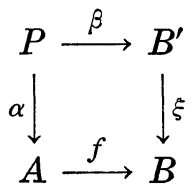

by setting $P=\operatorname{Ker} \nabla(f \oplus(-\xi))$ where $\nabla: B \oplus B \rightarrow B$ is the codiagonal map. We say that $\beta$ is the pullback of $f$ along $\xi$. Dually, we can construct pushouts. In an abelian category pushouts and pullbacks of kernels (cokernels) are kernels (cokernels). In pre-abelian categories only half of this is true.

THEOREM 1. Pullbacks of kernels are kernels. Specifically, if $f=\operatorname{ker} g$ and $\beta$ is the pullback of $f$ along $\xi$, then $\beta=\operatorname{ker} g \xi$. Dually, pushouts of cokernels are cokernels.

Proof. Let $\alpha$ be the pullback of $\xi$ along $f$ so we have the following diagram

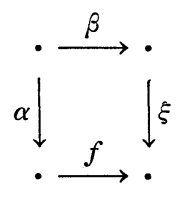

If $g \xi \lambda=0$, then $\xi \lambda=f \phi$ so, by the pullback property, there is a map $\theta$ such that $\beta \theta=\lambda$ (and $\alpha \theta=\phi$ ). To show that $\theta$ is unique, suppose $\beta \delta=0$. Then $\xi \beta \delta=f \alpha \delta=0$ so $\alpha \delta=0$ since $f$ is a kernel and hence monic. But $\beta \delta=0$ and $\alpha \delta=0$ imply that $\delta=0$ by the pullback property. 
Pushouts of kernels need not be kernels, or even monic. In the category of abelian $p$-groups with no elements of infinite height, the kernels are the $p$-adically closed subgroups. If $B$ is a direct sum of cyclic groups of order $p^{n}$ for $n=1,2,3, \cdots$ and $\bar{B}$ is the torsion subgroup of the corresponding product, and

$$
G[p]=\{g \in G: p g=0\},
$$

then the diagram

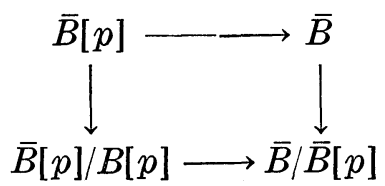

is a pushout in the category. The top row is a kernel while the bottom row is the zero map.

Definition. A kernel (cokernel) is said to be semi-stable if every pushout (pullback) is a kernel (cokernel).

Since a pushout of a pushout is a pushout, a pushout of a semistable kernel is a semi-stable kernel. The product of kernels need not be a kernel. In the example above $B[p] \rightarrow \bar{B}[p]$ is a kernel, and $\bar{B}[p] \rightarrow \bar{B}$ is a kernel, but the composite $B[p] \rightarrow \bar{B}$ is not a kernel. However we have the following.

THEOREM 2. The product of semi-stable kernels (cokernels) is $\alpha$ semi-stable kernel (cokernel).

Proof. Let $f: A \rightarrow B$ and $g: B \rightarrow C$ be semi-stable kernels. Consider the diagram

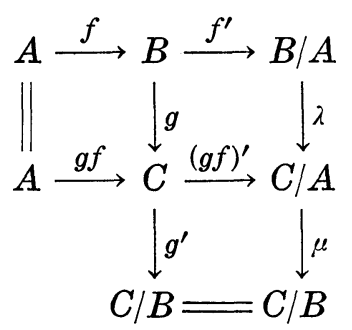

where $f^{\prime}, g^{\prime}$, and $(g f)^{\prime}$ are the cokernels of $f, g$, and $g f$. The upper right square is a pushout so $\lambda$ is a kernel. Suppose $(g f)^{\prime} \xi=0$. Then $g^{\prime} \xi=0$ so $\xi=g \phi$. But $\lambda f^{\prime} \phi=(g f)^{\prime} g \phi=(g f)^{\prime} \xi=0$ and $\lambda$ is monic (since it is a kernel) so $f^{\prime} \phi=0$ and hence $\phi=f \psi$ whereupon $\xi=g f \psi$. Since $g f$ is monic $\psi$ is unique whence $g f$ is a kernel of 
$(g f)^{\prime}$. Thus the composite of semi-stable kernels is a kernel.

To show that $g f$ is a semi-stable kernel consider the diagram

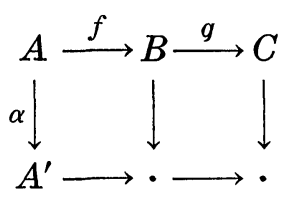

where the two squares are pushouts. Both bottom arrows are semistable kernels, so their composite is a kernel. But the composite is a pushout of $g f$ along $\alpha$. Hence $g f$ is a semi-stable kernel.

2. Stable exact sequences. A sequence $E$ is a diagram $A \stackrel{f}{\rightarrow} B \stackrel{g}{\rightarrow} C$ such that $g f=0$. A morphism between two sequences $E$ and $E^{\prime}$ is a triple $(\alpha, \beta, \gamma)$ of maps such that $f^{\prime} \alpha=\beta f$ and $g^{\prime} \beta=\gamma g$. We say that $E$ is left exact if $f$ is a kernel of $g$, right exact if $g$ is a cokernel of $f$, and exact if it is both left exact and right exact.

Suppose $E$ is a sequence and $\alpha: A \rightarrow A^{\prime}$ and $\beta: C^{\prime} \rightarrow C$. Then we can construct sequences $\alpha E$ and $E \beta$ as follows. To construct $\alpha E$ pushout $f$ along $\alpha$.

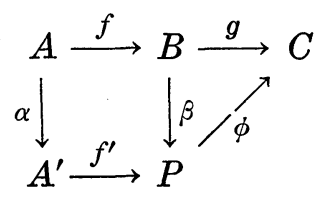

The map $\phi$ from $P$ to $C$ such that $\phi \beta=g$ and $\phi f^{\prime}=0$ exists and is unique by the property of pushouts. The sequence $A^{\prime} \rightarrow P \rightarrow C$ is denoted by $\alpha E$. We construct $E \beta$ dually.

LEMMA 3. The pushout diagram $E \rightarrow \alpha E$ is characterized by the property that any morphism $(\theta \alpha, \cdot, \cdot)$ from $E$ to $F$ factors through a unique morphism $(\theta, \cdot, \cdot)$ from $\alpha E$ to $F$. Dually, the pullback diagram $E \beta \rightarrow E$ is characterized by the property that any morphism $(\cdot, \cdot, \beta \theta)$ from $F$ to $E$ factors through a unique morphism $(\cdot, \cdot, \theta)$ from $F$ to $E \beta$.

Proof. Follows immediately from the universal properties of pushouts and pullbacks.

THEOREM 4. If $E$ is a sequence, then

$$
\begin{gathered}
\left(\alpha_{1} \alpha_{2}\right) E=\alpha_{1}\left(\alpha_{2} E\right) \\
\left(E \beta_{1}\right) \beta_{2}=E\left(\beta_{1} \beta_{2}\right) \\
(\alpha E) \beta=\alpha(E \beta)
\end{gathered}
$$


Proof. All three equalities follow easily by diagram chasing using the characterizations of the preceding lemma.

THEOREM 5. If $E$ is right exact, then $\alpha E$ is right exact. If $E$ is left exact, then $E \beta$ is left exact. Hence if $\alpha E$ is left exact and $E \beta$ is right exact, then $(\alpha E) \beta=\alpha(E \beta)$ is exact.

Proof. Let the situation $E \rightarrow \alpha E$ be written out as

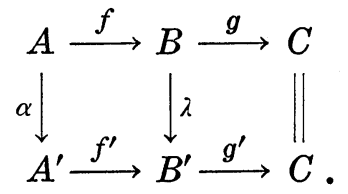

Suppose $\phi f^{\prime}=0$. Then $\phi f^{\prime} \alpha=0$ so $\phi \lambda f=0$. Hence $\phi \lambda=\theta g$ for a unique $\theta$. We shall show that $\phi=\theta g^{\prime}$. Now $\phi f^{\prime}=0=\theta g^{\prime} f^{\prime}$ and $\phi \lambda=\theta g=\theta g^{\prime} \lambda$, so $\phi-\theta g^{\prime}$ kills both $\lambda$ and $f^{\prime}$. But since $B^{\prime}$ is a pushout, we have $\phi-\theta g^{\prime}=0$. The second statement is proved dually. The third is immediate from the first two and Theorem 4.

Our objective is to define $\operatorname{Ext}(C, A)$. An element of Ext $(C, A)$ should be an exact sequence. Moreover we would like $\operatorname{Ext}(C, A)$ to be a functor. In abelian categories if $\alpha: A \rightarrow A^{\prime}$ we get a map from $\operatorname{Ext}(C, A)$ to $\operatorname{Ext}\left(C, A^{\prime}\right)$ by taking $E$ to $\alpha E$. In pre-abelian categories $\alpha E$ need not be exact even when $E$ is, as we saw in the category of abelian $p$-groups with no elements of infinite height. Accordingly we restrict our attention to those exact sequence that remain exact upon composition with maps.

Definition. An exact sequence $E$ is said to be a stable if $\alpha E$ and $E \beta$ are exact for all maps $\alpha$ and $\beta$. A map is said to be a stable kernel (cokernel) if it is the kernel (cokernel) map in a stable exact sequence.

If $E$ is exact, then $\alpha E$ is right exact by Theorem 5 . Hence if the kernel of $E$ is semi-stable, then $\alpha E$ is exact. Dually, if the cokernel of $E$ is semi-stable, then $E \beta$ is exact. So if the kernel and cokernel of $E$ are semi-stable, then $E$ is stable. Conversely if $E$ is stable, then its kernel and cokernel are clearly semi-stable. By Theorems 4 and 5 if $E$ is stable, then so are $\alpha E$ and $E \beta$.

3. The five lemma. The proof of the five lemma in an easy diagram chase if you have elements. In a pre-abelian category the notion of a stable cokernel allows this diagram chase to be carried out using pullbacks.

THEOREM 6. Let $E$ be an exact sequence with a semi-stable 
cokernel, and let $E^{\prime}$ be a left exact sequence. If $(1, \phi, 1): E \rightarrow E^{\prime}$, then $\phi$ is an isomorphism.

Proof. Consider the diagram

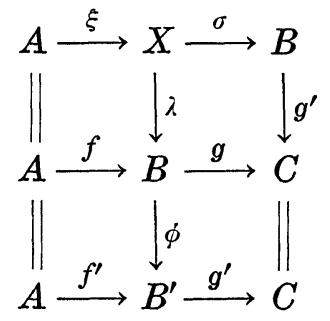

where the rows are $E g^{\prime}, E$ and $E^{\prime}$. Then $g^{\prime}(\phi \lambda-\sigma)=0$ so $\phi \lambda-\sigma=f^{\prime} \rho$ for some $\rho: X \rightarrow A$ since $f^{\prime}=\operatorname{ker} g^{\prime}$. We have $f^{\prime} \rho \xi=\phi \lambda \xi=f^{\prime}$ so $\rho \xi=1$ since $f^{\prime}$ is monic. Thus $(1-\xi \rho) \xi=0$ so there is a $\theta: B^{\prime} \rightarrow X$ such that $\theta \sigma=1-\xi \rho$ since $g$ is a semi-stable cokernel and hence $\sigma=$ coker $\xi$. In particular $\rho \theta \sigma=0$ and $\sigma \theta \sigma=\sigma$ so, since $\sigma$ is epic, we have $\rho \theta=0$ and $\sigma \theta=1$. Then $\phi \lambda \theta=$ $\left(f^{\prime} \rho+\sigma\right) \theta=1$. Moreover $\phi$ is monic for if $\phi \delta=0$ then $g \delta=g^{\prime} \phi \delta=0$ so $\delta=f \varepsilon$, since $f=\operatorname{ker} g$, and we have $0=\phi \delta=\phi f \varepsilon=f^{\prime} \varepsilon$ so $\varepsilon$, and hence $\delta$, is 0 . Thus $\phi \lambda \theta \phi=\phi$ so $\lambda \theta \phi=1$ and $\lambda \theta=\phi^{-1}$.

CoROllary 7. If $E$ and $F$ are exact, either $E$ or $F$ is stable, and $(\alpha, \cdot, \beta): E \rightarrow F$, then $\alpha E=F \beta$ in the sense that there is a map $(1, \phi, 1): \alpha E \rightarrow F \beta$ such that $\phi$ is an isomorphism.

Proof. By duality it suffices to consider the case where $E$ is stable. By Lemma 3 the map $(\alpha, \cdot, \beta): E \rightarrow F$ factors through a unique map $(1, \cdot, \beta): \alpha E \rightarrow F$ which factors through a unique map $(1, \cdot, 1): \alpha E \rightarrow F \beta$. Since $\alpha E$ is stable exact and $F \beta$ is left exact the conclusion follows from Theorem 6 .

The five lemma need not hold for exact sequences that are not stable. We will give an example where it fails, in the category of valuated abelian groups, at the end of the paper.

4. The functor Ext. We define $\operatorname{Ext}(C, A)$ to be the set of stable exact sequences $A \rightarrow \cdot \rightarrow C$ where two sequences are considered equal if there is a map $\left(1_{A}, \phi, 1_{C}\right)$ between them with $\phi$ an isomorphism. Then Theorems 4 and 5 imply that Ext $(C, A)$ is a bifunctor-covariant in $A$ and contravariant in $C$. To turn $\operatorname{Ext}(C, A)$ into an abelian group we define the usual Baer sum of two stable exact sequences $E_{1}$ and $E_{2}$ by

$$
E_{1}+E_{2}=\nabla\left(E_{1} \oplus E_{2}\right) \Delta
$$


where $\Delta: C \rightarrow C \oplus C$ is the diagonal map and $\nabla: A \oplus A \rightarrow A$ is the codiagonal map. The proof that this turns $\operatorname{Ext}(C, A)$ into an abelian group follows MacLane [3, pp. 70-71]. The only difficulty that is peculiar to the present situation is the stability of $E_{1} \oplus E_{2}$.

THEOREM 8. If $E_{1}$ and $E_{2}$ are stable exact sequences, then $E_{1} \oplus E_{2}$ is a stable exact sequence.

Proof. Let $E_{i}$ be $A_{i} \rightarrow B_{i} \rightarrow C_{i}$. The map $A_{1} \oplus A_{2} \rightarrow B_{1} \oplus A_{2}$ is a pushout of $A_{1} \rightarrow B_{1}$ and so is a semi-stable kernel. The map $B_{1} \oplus A_{2} \rightarrow B_{1} \oplus B_{2}$ is a pushout of $A_{2} \rightarrow B_{2}$ and so is a semi-stable kernel. By Theorem 2 the map $A_{1} \oplus A_{2} \rightarrow B_{1} \oplus B_{2}$ is a semi-stable kernel. Dually the map $B_{1} \oplus B_{2} \rightarrow C_{1} \oplus C_{2}$ is a semi-stable cokernel. Thus $E_{1} \oplus E_{2}$ is stable exact.

We now have all we need to follow the proof in [3, pp. 70-71] that $\operatorname{Ext}(C, A)$ is an abelian group, and in fact an additive bifunctor.

5. Products of stable maps. As in the case of proper monics in relative homological algebra in abelian categories, the key to proving exactness of the sequence of Ext's at one point is that the product of stable kernels (cokernels) is a stable kernel (cokernel).

THEOREM 9. If $f$ and $g$ are stable kernels, then $g f$ is a stable kernel.

Proof. Let $A \stackrel{f}{\rightarrow} B \stackrel{f^{\prime}}{\rightarrow} B / A$ and $B \stackrel{g}{\rightarrow} C \stackrel{g^{\prime}}{\rightarrow} C / B$ be stable exact sequences. Consider the diagram

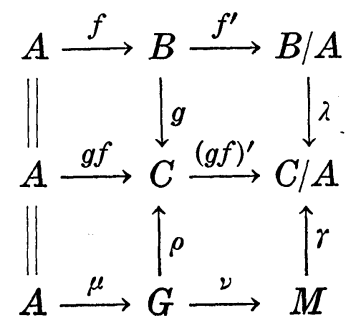

where $(g f)^{\prime}$ is a cokernel of $g f$ and the lower right hand square is a pullback. Since $g f$ is a semi-stable kernel it suffices to show that $\nu$ is the cokernel of $\mu$. Consider the diagram

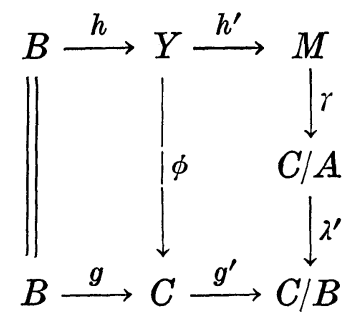


where $g^{\prime}$ and $\lambda^{\prime}$ are cokernels of $g$ and $\lambda$, and the right hand square is a pullback, so the top row is exact. There is a unique map $\varepsilon: G \rightarrow Y$ such that $\phi \varepsilon=\rho$ and $h^{\prime} \varepsilon=\nu$ since $Y$ is a pullback and $\lambda^{\prime} \gamma \nu=\lambda^{\prime}(g f)^{\prime} \rho=g^{\prime} \rho$. Consider the map from $Y$ to $C / A$ given by

$$
\gamma h^{\prime}-(g f)^{\prime} \dot{\phi} \text {. }
$$

Then $\lambda^{\prime} \gamma h^{\prime}-\lambda^{\prime}(g f)^{\prime} \dot{\phi}=g^{\prime} \phi-g^{\prime} \phi=0$ so $\gamma h^{\prime}-(g f)^{\prime} \phi=\lambda \theta$ because $\lambda$ is a kernel since $g$ is a stable kernel. Consider the pullback diagram

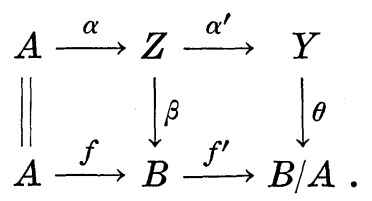

Define $\sigma: Z \rightarrow G$ by $\nu \sigma=h^{\prime} \alpha^{\prime}$ and $\rho \sigma=g \beta+\phi \alpha^{\prime}$. This is OK because $G$ is a pullback and

$$
(g f)^{\prime}\left(g \beta+\phi \alpha^{\prime}\right)=\lambda f^{\prime} \beta+(g f)^{\prime} \phi \alpha^{\prime}=\lambda \theta \alpha^{\prime}+(g f)^{\prime} \phi \alpha^{\prime}=\gamma h^{\prime} \alpha^{\prime} .
$$

Define $\sigma^{*}: G \rightarrow Z$ by $\alpha^{\prime} \sigma^{*}=\varepsilon$ and $\beta \sigma^{*}=0$. This is ok because $Z$ is a pullback and $\lambda \theta \varepsilon=\left(\gamma h^{\prime}-(g f)^{\prime} \phi\right) \varepsilon=\gamma \nu-(g f)^{\prime} \rho=0$ and $\lambda$ is a kernel so $\theta \varepsilon=0$. Since $\rho \sigma \sigma^{*}=\left(g \beta+\phi \alpha^{\prime}\right) \sigma^{*}=\phi \varepsilon=\rho$ and $\nu \sigma \sigma^{*}=$ $h^{\prime} \alpha^{\prime} \sigma^{*}=h^{\prime} \varepsilon=\nu$, we have $\sigma \sigma^{*}=1$.

Suppose $\xi: G \rightarrow W$ and $\xi \mu=0$. Then $\nu \sigma \alpha=h^{\prime} \alpha^{\prime} \alpha=0$ and $\rho \sigma \alpha=g \beta \alpha=g f$, so $\sigma \alpha=\mu$ so $\xi \sigma \alpha=0$ so $\xi \sigma=\tau \alpha^{\prime}$ for some $\tau: Y \rightarrow W$. Define $\beta^{*}: B \rightarrow Z$ by $\alpha^{\prime} \beta^{*}=-h$ and $\beta \beta^{*}=1$. This is ok because $-\theta h=f^{\prime}$ since $\lambda f^{\prime}=(g f)^{\prime} g=-\lambda \theta h$ and $\lambda$ is a kernel. So $\tau h=$ $-\tau \alpha^{\prime} \beta^{*}=-\xi \sigma \beta^{*}$. Now $\nu \sigma \beta^{*}=h^{\prime} \alpha^{\prime} \beta^{*}=0$ and $\rho \sigma \beta^{*}=g-\phi h=0$, so $\sigma \beta^{*}=0$ so $\tau h=0$ so $\tau=\delta h^{\prime}$ for some $\delta: M \rightarrow W$. It remains to show that $\delta \nu=\xi$. But $\delta \nu \sigma=\delta h^{\prime} \alpha^{\prime}=\tau \alpha^{\prime}=\xi \sigma$, so $\delta \nu=\delta \nu \sigma \sigma^{*}=\xi \sigma \sigma^{*}=\xi$. The map $\delta$ is unique because $\nu$ is epic since $\nu \sigma=h^{\prime} \alpha^{\prime}$.

We have the following converse to Theorem 9 .

Theorem 10. If $f g$ is a (stable) kernel, and $f$ is a semi-stable kernel, then $g$ is a (stable) kernel.

Proof. Consider the following diagram where $f^{\prime}, g^{\prime}$, and $(f g)^{\prime}$ are the cokernels of $f, g$, and $f g$.

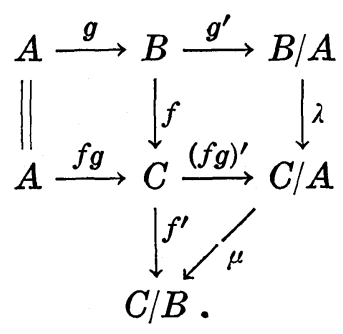


We shall show that the upper right square is a pullback, which proves the theorem by Theorems 4 and 5. Suppose $\lambda \phi=(f g)^{\prime} \psi$. Then $0=\mu \lambda \phi=\mu(f g)^{\prime} \psi=f^{\prime} \psi$, so $\psi=f \theta$ for a unique $\theta$. We must show that $g^{\prime} \theta=\phi$. But $\lambda g^{\prime} \theta=(f g)^{\prime} f \theta=(f g)^{\prime} \psi=\lambda \phi$ and $\lambda$ is a kernel since $f$ is a semi-stable kernel. Thus $g^{\prime} \theta=\phi$ and $B$ is a pullback.

6. The $3 \times 3$ lemma. The proof of the $3 \times 3$ lemma is another exercise in translating element chases to pullbacks.

THEOREM 11. If in the commutative diagram

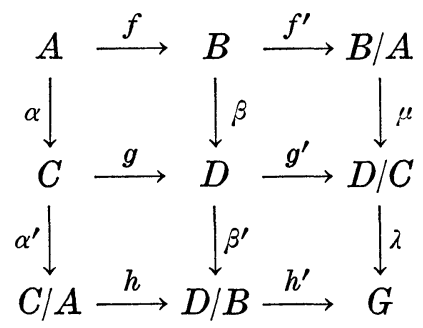

the rows and the first two columns are stable exact, then the last column is stable exact.

Proof. We first show that $\lambda=$ coker $\mu$. Now $\lambda g^{\prime}=h^{\prime} \beta^{\prime}$ is epic, so $\lambda$ is epic. Suppose $\xi \mu=0$. Then $\xi \mu f^{\prime}=\xi g^{\prime} \beta=0$ so $\xi g^{\prime}=\phi \beta^{\prime}$ for some $\phi$ since $\beta^{\prime}=$ coker $\beta$. Then $\phi h \alpha^{\prime}=\phi \beta^{\prime} g=\xi g^{\prime} g=0$ so $\phi h=0$ so $\phi=\psi h^{\prime}$ for some $\psi$ since $h^{\prime}$ is the cokernel of $h$. We must show that $\psi \lambda=\xi$. But $\psi \lambda g^{\prime}=\psi h^{\prime} \beta^{\prime}=\phi \beta^{\prime}=\xi g^{\prime}$ and $g^{\prime}$ is epic. So $\lambda=\operatorname{coker} \mu$.

Next we show that $\mu$ is monic by showing that ker $\mu=0$. Consider the pullback diagram

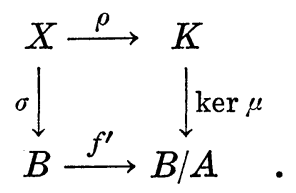

Since $\rho$ is epic, because $f^{\prime}$ is stable, it sufficies to show that $(\operatorname{ker} \mu) \rho=f^{\prime} \sigma=0$. Now $g^{\prime} \beta \sigma=\mu f^{\prime} \sigma=0$ so $\beta \sigma=g \tau$ for some $\tau: X \rightarrow C$. Thus $h \alpha^{\prime} \tau=\beta^{\prime} g \tau=\beta^{\prime} \beta \sigma=0$ so $\alpha^{\prime} \tau=0$ so $\tau=\alpha \theta$ for some $\theta: X \rightarrow A$. Hence $\beta \sigma=g \tau=g \alpha \theta=\beta f \theta$ which implies, since $\beta$ is monic, that $\sigma=f \theta$, whereupon $f^{\prime} \sigma=f^{\prime} f \theta=0$ and we have shown that $\mu$ is monic.

To show that $\mu=\operatorname{ker} \lambda$, suppose $\xi \lambda=0$ and consider the pullback diagram 


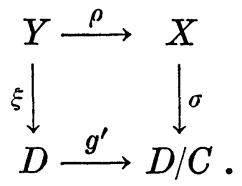

We have $h^{\prime} \beta^{\prime} \sigma=\lambda g^{\prime} \sigma=\lambda \xi \rho=0$ so $\beta^{\prime} \sigma=h \delta$ for some $\delta: Y \rightarrow C / A$. Consider the pullback diagram

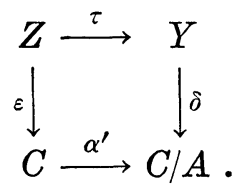

We have $\beta^{\prime} g \varepsilon=h \alpha^{\prime} \varepsilon=h \delta \tau=\beta^{\prime} \sigma \tau$, so $\beta^{\prime}(\sigma \tau-g \varepsilon)=0$ so $\sigma \tau-g \varepsilon=\beta \phi$ for some $\phi: Z \rightarrow B$. Now $\mu f^{\prime} \phi=g^{\prime} \beta \phi=g^{\prime} \sigma \tau$ kills $\operatorname{ker} \tau$ and $\mu$ is monic, so $f^{\prime} \phi=\nu \tau$ for some $\nu: Y \rightarrow B / A$ since $\tau$ is the pullback of the stable cokernel $\alpha^{\prime}$. Similarly $\mu \nu \tau=\mu f^{\prime} \phi=g^{\prime} \beta \phi=g^{\prime} \sigma \tau$, so $\mu \nu=g^{\prime} \sigma=\xi \rho$ kills $\operatorname{ker} \rho$, so $\nu=\eta \rho$ for some $\eta: X \rightarrow B / A$ since $\rho$ is a pullback of the stable cokernel $g^{\prime}$. But $\mu \eta \rho=\mu \nu=\xi \rho$ and $\rho$ is epic, so $\mu \eta=\xi$, and since $\mu$ is monic, $\eta$ is unique. This completes the proof that the last column is exact. Finally, $\lambda g^{\prime}=h^{\prime} \beta^{\prime}$ is a stable cokernel by Theorem 9 (or rather its dual) and hence $\lambda$ is a stable cokernel by the dual of Theorem 10 .

7. The long exact Ext sequences. We can define the groups $\operatorname{Ext}^{n}(C, A)$ for $n=0,1$ by letting $\operatorname{Ext}^{0}(C, A)=\operatorname{Hom}(C, A)$ and $\operatorname{Ext}^{1}(C, A)=\operatorname{Ext}(C, A)$. The elements of $\operatorname{Ext}^{n}(C, A)$ for $n>1$ are Yoneda composites

$$
E_{n} \circ E_{n-1} \circ \cdots \circ E_{1}
$$

where $E_{i} \in \operatorname{Ext}\left(X_{i}, X_{i+1}\right)$ and $X_{1}=C$ and $X_{n+1}=A$ subject to the usual equivalence relation (see [3, pp. 82-87]). There are no further problems in pursuing the usual treatment of "Ext without projectives." In particular the proof of Theorem 5.1 in [3] goes through giving us

Theorem 12. Let $E$ be a stable exact sequence $A \rightarrow B \rightarrow C$ in a pre-abelian category, and let $G$ be any object in that category. Then we get an exact sequence of abelian groups

$\operatorname{Ext}^{n-1}(B, G) \rightarrow \operatorname{Ext}^{n-1}(A, G) \rightarrow \operatorname{Ext}^{n}(C, G) \rightarrow \operatorname{Ext}^{n}(B, G) \rightarrow \operatorname{Ext}^{n}(A, G)$ for $n=1,2, \cdots$.

8. Relative theories. Quite often in abelian categories we are 
more interested in a relative homological algebra than in the absolute one. For example we may wish to study purity in abelian groups. The same holds true in pre-abelian categories, as we shall see in the case of topological abelian groups and valuated abelian groups. Instead of considering all sequences in $\operatorname{Ext}(C, A)$, we select a subset Pext $(C, A)$ of proper exact sequences such that

1. If $E \in \operatorname{Pext}(C, A)$ and $\alpha: A \rightarrow A^{\prime}$, then $\alpha E \in \operatorname{Pext}\left(C, A^{\prime}\right)$.

2. If $E \in \operatorname{Pext}(C, A)$ and $\beta: C^{\prime} \rightarrow C$, then $E \beta \in \operatorname{Pext}\left(C^{\prime}, A\right)$.

3. If $E_{1} \in \operatorname{Pext}\left(C_{1}, A_{1}\right)$ and $E_{2} \in \operatorname{Pext}\left(C_{2}, A_{2}\right)$, then $E_{1} \oplus E_{2}$ is in $\operatorname{Pext}\left(C_{1} \oplus C_{2}, A_{1} \oplus A_{2}\right)$.

4. If $A \subseteq B \rightarrow B / A$ and $B \subseteq C \rightarrow C / B$ are proper exact, then $A \subseteq C \rightarrow C / A$ is proper exact.

5. If Ker $f \rightarrow A \stackrel{f}{\rightarrow} B$ and Ker $g \rightarrow B \stackrel{g}{\rightarrow} C$ are proper exact, then Ker $g f \rightarrow A \stackrel{g f}{\rightarrow} C$ is proper exact.

A kernel (cokernel) is called proper if it is the kernel (cokernel) of a proper exact sequence. We could, equivalently, write down properties that must be satisfied by proper kernels (or proper cokernels). Note that 4 says that the product of proper kernels is a proper kernel, and 5 says that the product of proper cokernels is a proper cokernel. These five properties imply that $\operatorname{Pext}(C, A)$ is a subgroup of $\operatorname{Ext}(C, A)$. Actually, property 3 is redundant and can be derived from 1 and 4 , or 2 and 5 , as in the proof of Theorem 8. More useful is a remarkable theorem proved by Nunke [2] in abelian categories, that properties 4 and 5 are equivalent. The proof for pre-abelian categories follows Nunke's argument.

THEOREM 13. If products of proper cokernels are proper, then products of proper kernels are proper.

Proof. Suppose $A \stackrel{f}{\rightarrow} B \stackrel{f^{\prime}}{\rightarrow} B / A$ and $B \stackrel{g}{\rightarrow} C \stackrel{g^{\prime}}{\rightarrow} C / B$ are proper exact. We wish to show that the exact sequence $A \stackrel{g f}{\longrightarrow} C \stackrel{(g f)^{\prime}}{\longrightarrow} C / A$ is proper. The map $h: B / A \rightarrow C / A$ is a (proper) kernel since it is a pushout of $g: B \rightarrow C$. Let $\iota_{i}$ and $\pi_{i}$ be the injection and projection on the $i^{\text {th }}$ factor of $B / A \oplus C$ and consider the diagram

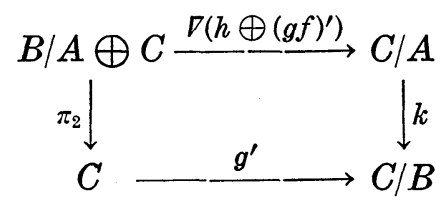

which commutes since $k \nabla\left(h \oplus(g f)^{\prime}\right)=\nabla(k \oplus k)\left(h \oplus(g f)^{\prime}\right)=\nabla\left(k h \oplus k(g f)^{\prime}\right)=\nabla\left(0 \oplus g^{\prime}\right)=g^{\prime} \pi_{2}$. 
We shall show that the diagram is a pullback. Suppose $g^{\prime} \phi=k \psi$. If we want a map $\lambda$ into $B / A \oplus C$ such that $\pi_{2} \lambda=\phi$ and $\nabla\left(h \oplus(g f)^{\prime}\right) \lambda=\psi$, then we must have

$$
\psi=\nabla\left(h \oplus(g f)^{\prime}\right)\left(\iota_{1} \pi_{1} \lambda+\iota_{2} \phi\right)=h \pi_{1} \lambda+(g f)^{\prime} \phi
$$

so we need $h \pi_{1} \lambda=\psi-(g f)^{\prime} \phi$. But $k\left(\psi-(g f)^{\prime} \phi\right)=k \psi-g^{\prime} \phi=0$ so such a map $\pi_{1} \lambda$ exists and is unique. Conversely, the same calculations show that if we define $\lambda$ by $\pi_{2} \lambda=\phi$ and $h \pi_{1} \lambda=\psi-(g f)^{\prime} \phi$, then $\pi_{2} \lambda=\dot{\phi}$ and $\nabla\left(h \oplus(g f)^{\prime}\right) \lambda=\psi$. Thus the diagram is a pullback.

The map $\left(f^{\prime} \oplus 1\right): B \oplus C \rightarrow B / A \oplus C$ is a proper cokernel since it is a pullback of $f^{\prime}$. Hence the map $B \oplus C \rightarrow C / A$ given by

$$
\nabla\left(h \oplus(g f)^{\prime}\right)\left(f^{\prime} \oplus 1\right)=\nabla\left(h f^{\prime} \oplus(g f)^{\prime}\right)=(g f)^{\prime} \nabla(g \oplus 1)
$$

is a proper cokernel, so $(g f)^{\prime}$ is a proper cokernel (it is an easy consequence of property 1 and the five lemma that if $\alpha \beta$ is a proper cokernel then so is $\alpha$ ).

The same comments made in the last section apply here, and we get Theorem 12 with $E$ a proper exact sequence and $E \mathrm{xt}^{n}$ replaced by Pext ${ }^{n}$.

9. Pre-abelian categories from radicals. One way pre-abelian categories arise is as full subcategories of abelian categories. Recall that a radical $R$ in an abelian category is a functorial subobject such that $R(A / R(A))=0$ for every object $A$.

THEOREM 14. If $R$ is a radical in an abelian category $\mathscr{A}$, then the full subcategory $\mathscr{S}$ of objects $A$ such that $R(A)=0$ is a preabelian category in which every cokernel is semi-stable.

Proof. If $f$ is a map in $\mathscr{S}$, then any kernel of $f$ in $\mathscr{A}$ is in $\mathscr{S}$ and serves as a kernel there. If $g: B \rightarrow C$ is a cokernel of $f$ in $\mathscr{A}$, then $h g$ is a cokernel of $f$ in $\mathscr{S}$ where $h$ is the map $C \rightarrow C / R(C)$. To see that cokernels are semi-stable it suffices to observe that $g$ is a cokernel in $\mathscr{S}$ if and only if $g$ is a cokernel in $\mathscr{A}$ whose domain and range are in $\mathscr{S}$, and that pullbacks in $\mathscr{S}$ are pullbacks when viewed in $\mathscr{A}$.

If we let $R$ be the radical $R(A)=p^{\omega} A=\cap p^{n} A$ in the category of abelian groups $\mathscr{A}$ we get the pre-abelian category $\mathscr{S}$ of abelian groups with no elements of infinite $p$-height. The exact sequences $A \rightarrow B \rightarrow C$ in $\mathscr{S}$ are simply those sequences in $\mathscr{S}$ which are exact in $\mathscr{A}$. Since every cokernel is semi-stable, Ext will be determined if we characterize the semi-stable kernels. 
THEOREM 15. Let $\mathscr{S}$ be the category of abelian groups with no elements of infinite p-height. Let $B$ be in $\mathscr{S}$ and let $A \subseteq B$ be a subgroup. Then $A$ is a semi-stable (and hence stable) kernel in $\mathscr{S}$ if and only if

(i) $A$ is p-adically closed in $B$, i.e., $B / A$ has no elements of infinite p-height, and

(ii) The p-adic topology on $A$ is the relative topology induced by the p-adic topology on $B$, i.e., for each $m$ there is an $n$ such that $A \cap p^{n} B \subseteq p^{m} A$.

Proof. Suppose (i) and (ii) hold and consider the pushout diagram

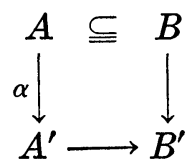

in the category of abelian groups, where $A^{\prime} \in \mathscr{S}$. It suffices to show that $B^{\prime} \in \mathscr{S}$. Now

$$
B^{\prime}=\left(A^{\prime} \oplus B\right) / K \quad \text { where } \quad K=\{(\alpha a,-a): a \in A\} .
$$

Suppose $\left(a^{\prime}, b\right) \in A^{\prime} \oplus B$ represents an element of infinite $p$-height in $B^{\prime}$. By (i) we may assume that $b$ has maximum $p$-height in $b+A$. If $b \neq 0$, then the $p$-height of $\left(a^{\prime}+\alpha a, b-a\right)$ is bounded, contradicting the assumption that $\left(a^{\prime}, b\right)$ represents an element of infinite $p$-height in $B^{\prime}$. Hence we may assume that $b=0$. So there must exist elements $a_{n} \in A$ such that $\left(a^{\prime}-\alpha a_{n}, a_{n}\right)$ has arbitrarily large height in $A^{\prime} \oplus B$. Hence $a_{n} \rightarrow 0$ in $B$, and therefore also in $A$ by (ii), while $\alpha a_{n} \rightarrow a^{\prime}$ in $A^{\prime}$. Thus $\alpha a_{n} \rightarrow 0$ and $\alpha a_{n} \rightarrow a^{\prime}$, whence $a^{\prime}=0$.

Conversely, suppose $A$ is a semi-stable kernel and $a_{n} \rightarrow 0$ in $B$. We must show that $a_{n} \rightarrow 0$ in $A$. Let $\alpha: A \rightarrow Z\left(p^{m}\right)$, where $Z\left(p^{m}\right)$ is the cyclic group of order $p^{m}$, and consider the pushout diagram

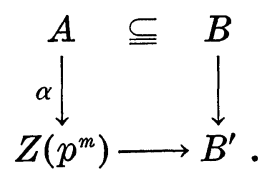

Then $\alpha\left(a_{n}\right)=0$ eventually, for otherwise we would have $0 \neq t \in Z\left(p^{m}\right)$ such that $t=\alpha\left(\alpha_{n}\right)$ for infinitely many $n$, giving $t$ infinite height in $B^{\prime}$. So $a_{n}$ must be eventually in $p^{m} A$ (or we could find a bad $\alpha$ ). Since this holds for arbitrary $m$, we have $a_{n} \rightarrow 0$ in $A$.

10. Topological abelian groups. Another way in which preabelian categories arise is by imposing additional structure on the 
objects of an abelian category. In the category of (Hausdorff) topological abelian groups the kernel of a map $f: A \rightarrow B$ is its group kernel, and the cokernel of $f$ is gotten by dividing $B$ by the closure of $f(A)$ and imposing the quotient topology. Thus kernels are precisely the closed subgroups and cokernels are the onto maps that are open (since the map onto a quotient group with the quotient topology is open).

THEOREM 16. In the category of topological abelian groups every exact sequence is stable.

Proof. Let $A \rightarrow B \rightarrow C$ be an exact sequence, let $\alpha: A \rightarrow A^{\prime}$ and consider the diagram

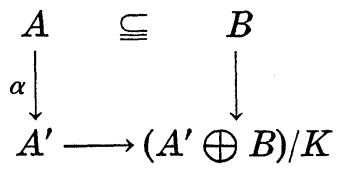

where $K=\{(\alpha \alpha,-\alpha): \alpha \in A\}$. To show that this is a pushout it suffices to prove that $K$ is closed in $A^{\prime} \oplus B$ and hence $\left(A^{\prime} \oplus B\right) / K$, with the quotient topology from $A^{\prime} \oplus B$, is Hausdorff. Suppose $\left(a^{\prime}, b\right) \notin K$. Then, if $b \notin A$, we have $\left(a^{\prime}, b\right) \in A^{\prime} \oplus(B \backslash A)$ which is an open set disjoint from $K$. If $b \in A$, then $-\alpha b \neq a^{\prime}$ so we can find disjoint open sets $U^{\prime}$ and $V^{\prime}$ in $A^{\prime}$ so that $a^{\prime} \in U^{\prime}$ and $-\alpha b \in V^{\prime}$. Then $-\alpha^{-1} V^{\prime}=A \cap V$ for some open set $V$ in $B$. So $\left(a^{\prime}, b\right)$ is in the open set $U^{\prime} \oplus V$ which is disjoint from $K$ for if $v \in V \cap A$ then $\alpha(-v) \in V^{\prime}$ which is disjoint from $U^{\prime}$.

To show that $A \subseteq B$ is a semi-stable kernel we must show that $A^{\prime}$ is imbedded homeomorphically as a closed subset of $\left(A^{\prime} \oplus B\right) / K$. It is certainly mapped one-to-one and continuously into $\left(A^{\prime} \oplus B\right) / K$. Moreover its image is the kernel of the induced map $\left(A^{\prime} \oplus B\right) / K \rightarrow B / A$ so it is a closed subset. To show that the imbedding is a homeomorphism, suppose $U \subseteq A^{\prime}$ is an open set containing zero. Choose an open set $V \cong A^{\prime}$ such that $V+V \subseteq U$ and $0 \in V$. Then $\alpha^{-1} V$ is open is $A$ and so $\alpha^{-1} V=A \cap W$ for some open set $W$ in $B$. Now $(V \oplus W) / K$ is an open set in $\left(A^{\prime} \oplus B\right) / K$ containing zero. We claim that $A^{\prime} \cap((V \oplus W) / K) \subseteq U$. In fact if $(v, w)+(\alpha \alpha,-\alpha)=(v+\alpha \alpha, 0)$, then $w=a$ so $\alpha a \in V$ so $v+\alpha a \in U$.

To show that the cokernel $\theta: B \rightarrow C$ is semi-stable, let $\gamma: C^{\prime} \rightarrow C$ and consider the pullback diagram

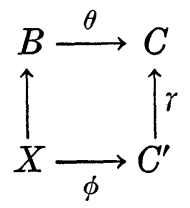


where $X=\left\{\left(b, c^{\prime}\right) \in B \oplus C^{\prime}: \theta b=\gamma c^{\prime}\right\}$. To show that $\phi$ is an open map, suppose $U \subseteq X$ is an open set containing zero. Then there exist open sets $V \subseteq B$ and $W \cong C^{\prime}$ containing zero such that $(V \oplus W) \cap X \subseteq U$. We may assume that $\gamma W \subseteq \theta V$ since $\theta V$ is open. Thus $W \subseteq \phi U$.

The locally compact abelian groups form a full subcategory of the topological abelian groups that is closed under kernels, cokernels, and extensions. Hence the exact sequences in this category are also all stable and of the form $A \subseteq B \rightarrow B / A$ where $A$ is closed in $B$ and $B \rightarrow B / A$ is an open map. Moskowitz [4] studied this set-up, determining the projectives and injectives. With these he defined Ext via resolutions for those groups that had them, which are very few. The theory we present defines $\operatorname{Ext}^{n}(C, A)$ in a natural way for any pair of locally compact abelian groups and any $n$. It is an easy consequence of the exact sequence of Ext's that this agrees with the resolution definition whenever resolutions exist.

Back in the category of topological abelian groups we obtain a relative theory if we consider the class of exact sequences $A \rightarrow B \rightarrow C$ which split as topological spaces in the sense that the map $B \rightarrow C$ admits a continuous cross-section. It is easily checked that these sequences form a proper class. This is the natural setting for free topological abelian groups which are projective here and allow one to define the relative $\mathrm{Ext}^{n}$ in terms of projective resolutions. Nummela [1] uses these resolutions to determine that the relative projective dimension of a compact abelian group is 1 .

11. Valuated abelian $p$-groups. This was the motivating example for the theory presented in this paper. Let $p$ be a fixed prime. Then a valuation $v$ on an abelian $p$-group $G$ assigns to each $x \in G$ an ordinal number $v x$, or the symbol $\infty$, such that

(1) $v(x+y) \geqq \min (v x, v y)$

(2) $v p x>v x$

(3) $v u x=v x$ if $p$ does not divide $u$.

Such objects arise naturally in abelian group theory as subgroups $G \subseteq H$ where $v$ is the height function on $H$ restricted to $G$. The category of valuated abelian groups will be the subject of a forthcoming paper by the authors. We state here, without proof, some of the results of that paper.

(1) A map $A \rightarrow B$ is a stable kernel if and only if $A$ is a subgroup of $B$ with the induced valuation, and every coset of $A$ contains an element of maximum value.

(2) A map $B \rightarrow C$ is a semi-stable cokernel if and only if $B$ maps onto $C$ and every element $c$ of $C$ comes from an element in $B$ whose value is arbitrarily close to the value of $c$ (in particular if 
the value of $c$ is $\infty$ or a nonlimit, then it must come from an element of the same value).

The category of valuated $p$-groups provides an example of the failure of the five lemma for exact sequences. Let $G$ be a $p$-group such that $p^{\omega} G$ is not divisible, and let $B$ be a basic subgroup of $G$. Let $H$ be the valuated group gotten from $G$ by setting $v x$ equal to the height of $x$ if $x \notin p^{\omega} G$ and $v x=\infty$ if $x \in p^{\omega} G$. Then we have the five lemma set-up

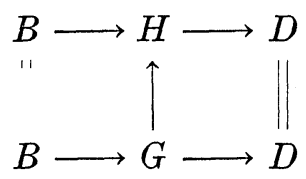

where $D$ is the divisible group $G / B$. But $G$ and $H$ are clearly not isomorphic as valuated groups.

\section{REFERENCES}

1. Eric C. Nummela, The progective dimension of a compact abelian group, Proc. Amer. Math. Soc., 38 (1973), 452-456.

2. R. J. Nunke, Purity and Subfunctors of the Identity, Topics in Abelian Groups, Scott, Foresman and Co., 1963.

3. Saunders MacLane, Homology, Academic Press, 1963.

4. Martin Moskowitz, Homological algebra in locally compact abelian groups, Trans. Amer. Math. Soc., 127 (1967), 361-404.

Received October 29, 1976. This research was supported by the National Science Foundation grant MPS71-02778 A04.

New Mexico State University

LAS CRUCES, NM 88003 



\section{PACIFIC JOURNAL OF MATHEMATICS}

\section{EDITORS}

RICHARD ARENS (Managing Editor)

University of California

Los Angeles, CA 90024

Charles W. Curtis

University of Oregon

Eugene, OR 97403

C. C. MOORE

University of California

Berkeley, CA 94720

\section{J. DugundJI}

Department of Mathematics

University of Southern California

Los Angeles, CA 90007

R. FinN and J. Milgram

Stanford University

Stanford, CA 94305

ASSOCIATE EDITORS
E. F. BECKENBACH
B. H. NeumanN
F. WOLF
K. YOSHIDA

\section{SUPPORTING INSTITUTIONS}

UNIVERSITY OF BRITISH COLUMBIA CALIFORNIA INSTITUTE OF TECHNOLOGY

UNIVERSITY OF CALIFORNIA

MONTANA STATE UNIVERSITY

UNIVERSITY OF NEVADA, RENO

NEW MEXICO STATE UNIVERSITY

OREGON STATE UNIVERSITY

UNIVERSITY OF OREGON

OSAKA UNIVERSITY

\author{
UNIVERSITY OF SOUTHERN CALIFORNIA \\ STANFORD UNIVERSITY \\ UNIVERSITY OF HAWAII \\ UNIVERSITY OF TOKYO \\ UNIVERSITY OF UTAH \\ WASHINGTON STATE UNIVERSITY \\ UNIVERSITY OF WASHINGTON \\ AMERICAN MATHEMATICAL SOCIETY
}

The Supporting Institutions listed above contribute to the cost of publication of this Journal, but they are not owners or publishers and have no responsibility for its content or policies.

Mathematical papers intended for publication in the Pacific Jaurnal of Mathematics should be in typed form or offset-reproduced, (not dittoed), double spaced with large margins. Please do not use built up fractions in the text of your manuscript. You may however, use them in the displayed equations. Underline Greek letters in red, German in green, and script in blue. The first paragraph or two must be capable of being used separately as a synopsis of the entire paper. Items of the bibliography should not be cited there unless absolutely necessary, in which case they must be identified by author and Journal, rather than by item number. Manuscripts, in triplicate, may be sent to any one of the editors. Please classify according to the scheme of Math. Reviews, Index to Vol. 39. All other communications should be addressed to the managing editor, or Elaine Barth, University of California, Los Angeles, California, 90024.

The Pacific Journal of Mathematics expects the author's institution to pay page charges, and reserves the right to delay publication for nonpayment of charges in case of financial emergency.

100 reprints are provided free for each article, only if page charges have been substantially paid. Additional copies may be obtained at cost in multiples of 50 .

The Pacific Journal of Mathematics is issued monthly as of January 1966. Regular subscription rate: $\$ 7200$ a year (6 Vols., 12 issues). Special rate: $\$ 36.00$ a year to individual members of supporting institutions.

Subscriptions, orders for back numbers, and changes of address should be sent to Pacific Journal of Mathematics, 103 Highland Boulevard, Berkeley, California, 94708.

PUBLISHED BY PACIFIC JOURNAL OF MATHEMATICS, A NON-PROFIT CORPORATION

Printed at Kokusai Bunken Insatsusha (International Academic Printing Co., Ltd.). 8-8, 3-chome, Takadanobaba, Shinjuku-ku, Tokyo 160, Japan.

Copyright (C) 1975 by Pacific Journal of Mathematics Manufactured and first issued in Japan 


\section{Pacific Journal of Mathematics}

\section{Vol. 71, No. $2 \quad$ December, 1977}

Krishnaswami Alladi and Paul Erdős, On an additive arithmetic

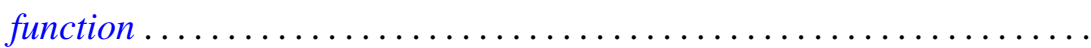

James Bailey and Dale Rolfsen, An unexpected surgery construction of a

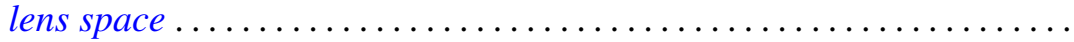

Lawrence James Brenton, On the Riemann-Roch equation for singular

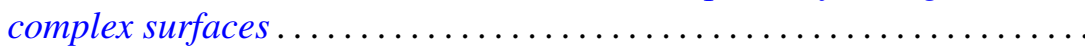

James Glenn Brookshear, Projective ideals in rings of continuous

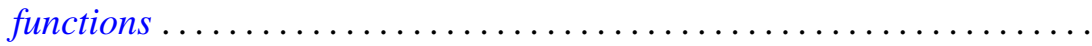

Lawrence Gerald Brown, Stable isomorphism of hereditary subalgebras of

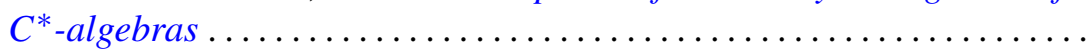

Lawrence Gerald Brown, Philip Palmer Green and Marc Aristide Rieffel, Stable isomorphism and strong Morita equivalence of $C^{*}$-algebras....

N. Burgoyne, Robert L. Griess, Jr. and Richard Lyons, Maximal subgroups

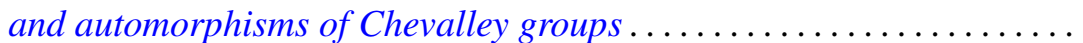
365

Yuen-Kwok Chan, Constructive foundations of potential theory .... 405

Peter Fletcher and William Lindgren, On $w \Delta$-spaces, $w \sigma$-spaces and

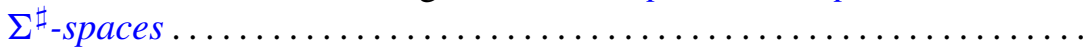

Louis M. Friedler and Dix Hayes Pettey, Inverse limits and mappings of

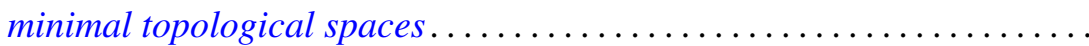

Robert E. Hartwig and Jiang Luh, A note on the group structure of unit regular ring elements.

I. Martin (Irving) Isaacs, Real representations of groups with a single involution ...

Nicolas P. Jewell, The existence of discontinuous module derivations . .

Antonio M. Lopez, The maximal right quotient semigroup of a strong semilattice of semigroups .......................

Dennis McGavran, $T^{n}$-actions on simply connected $(n+2)$-manifolds

Charles Anthony Micchelli and Allan Pinkus, Total positivity and the exact $n$-width of certain sets in $L^{1}$.

Barada K. Ray and Billy E. Rhoades, Fixed point-theorems for mappings with a contractive iterate .......................

Fred Richman and Elbert A. Walker, Ext in pre-Abelian categories. .

Raymond Craig Roan, Weak* generators of $H^{\infty}$ and $l^{1}$..

Saburou Saitoh, The exact Bergman kernel and the kernels of Szegö type...

Kung-Wei Yang, Operators invertible modulo the weakly compact 